\title{
On the Role of Recognition in Decision Making
}

\author{
Ben R. Newell and David R. Shanks \\ University College London
}

\begin{abstract}
In 2 experiments, the authors sought to distinguish between the claim that recognition of an object is treated simply as a cue among others for the purposes of decision making in a cue-learning task from the claim that recognition is attributed a special status with fundamental, noncompensatory properties. Results of both experiments supported the former interpretation. When recognition had a high predictive validity, it was relied on (solely) by the majority of participants; however, when other cues in the environment had higher validity, recognition was ignored, and these other cues were used. The results provide insight into when, where, and why recognition is used in decision making and also question the elevated status assigned to recognition in some frameworks (e.g., D. G. Goldstein \& G. Gigerenzer, 2002).
\end{abstract}

Observing that people can and do use recognition in making decisions is trivial. We can all think of many situations in which, when we are making "snap" decisions, or decisions in which the consequences of our actions will not be too great (e.g., choosing a breakfast cereal or a jar of peanut butter), we are quite happy to use simple recognition of a brand name to guide our decisions (Hoyer $\&$ Brown, 1990). We have known for 30 years that people utilize the availability of information to memory as a heuristic for judging the frequency of events and outcomes (Tversky \& Kahneman, 1973). Recognition is, presumably, a special case of availability because, at the extreme point of the availability continuum, an object does not come to mind at all because it is not recognized. By the same token, though, we can also imagine situations in which the potential consequences of a decision might lead us to consider more information than is provided by simple recognition or availability of an answer (e.g., a consumer buying a new computer or a stockbroker investing millions of dollars of clients' money).

Despite these intuitions about the way in which recognition is used in decision making, there is relatively little research directly evaluating the influence of recognition-based information. One notable exception is investigations of the recognition heuristic (Goldstein \& Gigerenzer, 2002), a recently proposed approach that places a very strong emphasis on the role of recognition in decision making. In this article, we examine the influence of recognitionbased information in a simple decision-making task and in so doing, attempt to (a) provide insight into when, where, and why

Ben R. Newell and David R. Shanks, Centre for Economic Learning and Social Evolution, University College London, London, United Kingdom.

We acknowledge the support of the Economic and Social Research Council (ESRC) and The Leverhulme Trust. The work was part of the program of the ESRC Research Centre for Economic Learning and Social Evolution. We thank Nicola Weston for help in collecting the data for Experiment 1, and David Lagnado, Mark Johansen, Magda Osman, Denis Hilton, Gerd Gigerenzer, and Danny Oppenheimer for helpful comments and discussions of drafts of this article.

Correspondence concerning this article should be addressed to Ben R. Newell, who is now at the School of Psychology, University of New South Wales, Sydney 2052, Australia. E-mail: ben.newell@unsw.edu.au recognition is used and (b) scrutinize the claims made by Goldstein and Gigerenzer (2002) concerning the influence of recognition.

The recognition heuristic states that when only one of two objects is recognized, then infer that the recognized alternative has the higher value with respect to the criterion being judged. There are certain caveats to this claim: The heuristic can only be applied usefully in domains in which (a) some (but not all) objects are unrecognized and (b) the recognition validity (i.e., the predictive validity of the recognition information) is higher than chance (.50).

There are at least two interpretations of how the recognition heuristic is intended to operate. One is that we rely on recognition when we have no other information and no possibility of obtaining further information to include in our decisions. If that is the case, then the claim is not so bold-any method, from utility maximizing to unit weighting to "one-reason" decision making, would lead to the same prediction. If all that is, or could be, available is one piece of information-in other words, the recognition of one object (or, conversely, not recognizing one object), then we should rely on it to make an inference, regardless of the mechanism underlying that decision process.

However, this does not seem to be the intended interpretation. Goldstein and Gigerenzer (2002) stated that the recognition heuristic is used in a noncompensatory fashion. Even when other information about a recognized alternative can be obtained, it never overrides the weight placed on simple recognition:

\footnotetext{
The recognition heuristic is a noncompensatory strategy: If one object is recognized and the other is not, then the inference is determined [italics added]; no other information about the recognized object is searched for and, therefore, no other information can reverse the choice determined by recognition. (Goldstein \& Gigerenzer, 2002, p. 82)
}

The intuition that when we recognize one object but not another we make an inference solely on the basis of recognition, with no possibility of it being overridden by other information, is a very strong claim to make.

What, then, is the basis for it? Supporting evidence comes principally from an empirical investigation of Goldstein and Gigerenzer's (2002) drosophila environment-the German cities task, an environment comprising the 83 largest cities in Germany 
and associated information (or cues) relating to different aspects of these cities (Do the cities have airports, football teams, universities?, etc.) in which participants are asked to choose the largest city from a series of paired alternatives. The evidence for the use of recognition in this domain is impressive, and the formulation of the recognition heuristic makes some sense for this environment. Recognition is a plausible predictor for city size, because we tend to hear about big cities and not small cities (though see Oppenheimer, 2003). However, it is not clear how far the results from one domain (city-size judgment) can be extrapolated as providing evidence for the existence of a recognition heuristic as a "cognitive adaptation" (Goldstein \& Gigerenzer, 2002, p. 88).

We return to the German cities environment in the General Discussion, but first, our aim is to reach further understanding of the psychological properties of recognition information by conducting an empirical examination of the use of recognition in a different domain - a simple cue-learning task. Specifically, we aim to determine whether recognition information is attributed an elevated status in cue-learning tasks such that it cannot be overridden by information carried by other cues in the environment.

The impetus for this examination is, first, a desire to know more about how recognition information is used in decision making, and second, a reaction to the emphasis placed on the information conveyed by recognition in the "fast-and-frugal" framework (Gigerenzer, Todd, and The ABC Research Group, 1999). Gigerenzer et al. conceive the mind as an "adaptive toolbox" containing a number of heuristics specified by simple search, stopping, and decision rules (e.g., take-the-best, minimalist, QuickEST; see Gigerenzer et al., 1999). Recognition plays a pivotal role in the approach because the use of recognition, or more specifically, the lack of recognition, exemplifies the ecological rationality of the heuristics contained in the adaptive toolbox. Ecological rationality is defined as the study of the match between heuristics and environmental structures (Gigerenzer, 2001).

Researchers have argued for the power and ecological rationality of recognition on the basis of ingenious simulations, which demonstrate that under certain circumstances, people who know more about a particular environment (e.g., German city populations) exhibit lower inferential accuracy than do people who know less $(60 \%$ vs. $68 \%$ correct in judging which of a pair of cities is larger—see Goldstein \& Gigerenzer, 2002, p.79, for a discussion of the basis of this effect). This "less is more" effect epitomizes the main thesis of the fast-and-frugal approach-namely, that the emphasis on speed and frugality replaces the methods of classical rationality (e.g., expected-utility theory or Bayesian reasoning) with "simple, plausible psychological mechanisms of inference .... that a mind can actually carry out under limited time and knowledge" (Gigerenzer \& Goldstein, 1996, p. 652).

The seductive appeal of heuristics that are simple, plausible, and powerful has made them very popular in the literature, and they have been applied to the analysis of many decision-making situations (e.g., Dhami \& Ayton, 2001; Elwyn, Edwards, Eccles, \& Rovner, 2001; Seeley, 2001). In spite of their popularity and emphasis on plausibility, however, evidence that people use the specific search, stopping, and decision rules that comprise the heuristics has been equivocal (Bröder, 2000, 2003; Bröder \& Schiffer, 2003; Chater, Oaksford, Nakisa, \& Redington, 2003; Juslin, Jones, Olsson \& Winman, 2003; Newell, Rakow, Weston,
\& Shanks, 2004; Newell \& Shanks, 2003; Newell, Weston, \& Shanks, 2003; Oppenheimer, 2003).

Rather than test the empirical basis for a particular heuristic, as we have done previously, in this article, we take a different approach by examining the plausibility of attributing recognition information the status that it has in the toolbox. We are interested in seeing whether the notion that recognition has a fundamental, noncompensatory, influence on people's choice behavior carries over to laboratory-based cue-learning tasks.

We had a number of reasons for using cue-learning tasks to examine the role of recognition. First, to our knowledge, it was the first time that recognition-based cues had been used in a cuelearning task. Second, we could use an environment in which we were able to carefully control the validity of both recognition information and other cues in the environment (something that is much harder to do in investigations of the role of recognition in many other tasks - e.g., brand recognition in consumer choice; see Hoyer \& Brown, 1990, or, indeed, the German cities task). Third, participants could learn about the properties of cues incrementally on a trial-by-trial basis, allowing us to conduct a fine-grained analysis of how recognition information affects participants' choices. Fourth, we could use a task for which we had a good deal of prior evidence that participants can learn about the properties of cues and can learn to make appropriate decisions in different environments (Bröder, 2000; Newell \& Shanks, 2003; Newell et al., 2003).

What could we hope to learn from such an investigation of recognition-based information? First, it would allow for theoretical development in considering the types of environments in which people rely on recognition information. Would recognition only work in domains in which there was a strong a priori belief about the relevance of recognition for the decision at hand (e.g., city size)? Or would recognition jump out and capture attention, regardless of the domain? Is it the case that relying on recognition only works when search is in memory and the attributes of the various objects are not immediately apparent in the external world (e.g., color, size, taste)? In consumer choice, it is interesting to note that selection of items on the basis of pure brand recognition reduces as participants have a chance to discover more about particular products (e.g., taste of a food item; Hoyer \& Brown, 1990). Such results imply that recognition exhibits the same properties as other predictors in a given domain-in other words, it is a cue, among others, that can be learned about and relied on if and when it is appropriate. Second, we could mark some boundary conditions for principles underpinning the fast-and-frugal approach and hope to determine when ignorance-based decision making flourishes or fails. Discovering such boundary conditions may not undermine the claims made for the recognition heuristic in the environments in which it has been empirically tested (e.g., the German cities environment), but it would serve to sharpen our thinking with regard to bold assertions about the adaptive, fundamental, and noncompensatory influence of recognition.

\section{Overview and Design of Experiment 1}

Our aim in Experiment 1 was to examine whether participants treated recognition information in a qualitatively different way than they treated the information provided by other cues in a cue-learning task. To examine this question, we used a stock 
market prediction game in which participants were presented with a series of two-alternative forced-choice investment decisions between two fictional companies. To induce recognition, we repeated a small number of company names and paired them with nonrepeated names in the hope that participants would learn to recognize the repeated names as the experiment progressed. We reasoned that recognizing the name of one company but not the other on a particular trial would allow participants to rely solely (if they so chose) on recognition in making their investment decisions.

In addition to the recognition information provided by the company name, participants could purchase investment advice from three financial advisors. These advisors provided binary information about whether to invest $(Y E S)$ or not to invest $(N O)$ in each company. Each cue had a validity and a discrimination rate. The validity of a cue is the probability that the cue identifies the correct alternative on a random selection of alternatives that differ on this cue. The discrimination rate is the proportion of occasions on which a cue has different values for each alternative. Newell et al. (2004) demonstrated that a function of validity and discrimination rate termed success (cf. Martignon \& Hoffrage, 1999) drove participants' search patterns in an environment similar to the one used here. They showed that a cue with the highest success rate in an environment was selected first (on average) and was rated most useful by the majority of participants. Thus, for Experiment 1, we created conditions in which the validity and discrimination rates of cues were varied to ensure that recognition information had either the highest or the lowest success rate. ${ }^{1}$ In the recognition high condition ( $\mathrm{RH})$, the company-name cue had the highest validity and the highest discrimination rate, making it the most successful piece of information for making correct investment decisions. In the recognition low condition (RL), the company-name cue was the least valid and least successful cue in the environment. Finally, in the no recognition condition (NR), we replaced the companyname cue with a fourth financial advisor, who gave his advice for free. This advice had the highest validity and the highest discrimination rate, making it the most successful piece of advice for making correct decisions. We included this third condition to provide an environment in which the informational properties (validity and discrimination rate) of the most successful cue were identical to the company-name cue in the RH condition, but in which this information was no longer associated with recognition.

Given this design, we made the predictions outlined in Table 1. If recognition information has special status-if, in other words, it

Table 1

Hypotheses and Predictions for Experiment 1

\begin{tabular}{ccc}
\hline & \multicolumn{2}{c}{ Predictions } \\
\cline { 2 - 3 } & $\begin{array}{c}\text { Proportion of } \\
\text { trials on which } \\
\text { recognized } \\
\text { company chosen }\end{array}$ & $\begin{array}{c}\text { Proportion of } \\
\text { trials on which } \\
\text { advice is } \\
\text { purchased }\end{array}$ \\
\hline \multicolumn{1}{c}{ Hypotheses } & $\mathrm{RH}=\mathrm{RL}$ & $\mathrm{RH}=\mathrm{RL}<\mathrm{NR}$ \\
$\begin{array}{l}\text { Status of recognition information } \\
\begin{array}{l}\text { Special } \\
\text { Consistent with other cues }\end{array} \\
\mathrm{RH}>\mathrm{RL}\end{array}$ & $\mathrm{NR}=\mathrm{RH}<\mathrm{RL}$ \\
\hline
\end{tabular}

Note. $\mathrm{RH}=$ recognition high $\mathrm{RL}=$ recognition low $\mathrm{NR}=$ no recognition. is used in noncompensatory way with no regard for the properties of other cues in the environment - then on all the trials on which participants recognize one company but not the other, they should choose to invest in the recognized company. Crucially, this proportion should not differ between the RH and RL conditions. In addition, on these trials, participants should not purchase any further information (advice), because recognition, if it is special, should be enough on its own for the decision. Thus, in the RH and RL conditions, purchase of advice should be of a similarly low proportion. We might also expect to see a somewhat higher proportion of advice purchased in the NR condition, because even though the provided information has the same informational properties, it is not associated with recognition and therefore might be attributed less importance.

In contrast, if recognition information is treated in a way consistent with other cues in the environment, then we expect the company-name cue to be assigned less importance when its validity and discrimination rate are low (RL), leading to fewer trials on which the recognized company is chosen and more trials on which advice is purchased. In addition, because the informational properties of the free advice and company-name cue are identical, we predicted that advice would be purchased on an equally small proportion of trials in the NR and RH conditions.

\section{Method}

Participants. Thirty-six members ( 23 women and 13 men, mean age $=$ 26 years) of the University College London community participated in the experiment in return for performance-related remuneration. Participants were assigned in equal numbers to the RH, RL, and NR groups.

Stimuli and design. The experiment was run on computers. Participants were presented with a series of two-alternative forced-choice investment decisions between two fictional companies. For each decision, participants in the two recognition conditions were provided (at no cost) with the names of the two companies. The novel company names were taken from a variety of nonword databases (e.g., the ARC Nonword Database; Rastle, Harrington, \& Coltheart, 2002); examples included $A B U B A$, $B L A U D S$, and FILZEC. The four company names that were repeated to induce recognition were ELBONICS, AGRAJET, MENTIFEX, and $H A X O R$. Three further pieces of information about each company were available at a cost to participants. These were the recommendations of three financial advisors (Richard, Tom, and Henry) to invest (YES) or not to invest $(N O)$ in the companies. In the NR condition, the company-name cue was replaced with free advice from a financial advisor named John.

Each piece of information or cue was assigned a validity and a discrimination rate. In each condition, the most valid piece of information (i.e. company-name cue for RH, Advisor 1 for RL, and free advice for NR) had a validity of .80 . The remaining advisors in the RH and NR conditions were assigned validities of $.75, .70$, and .65 , and in the RL condition, Advisors 2 and 3 were assigned .75 and .70, with company name taking .65. For each company, there were 16 distinct cue patterns ( 00000 through 111111 ). For each pattern, a 0 represents a $N O$ or a nonrepeated company name and a 1 represents a $Y E S$ or a repeated company name. Thus the pattern 10011 would appear on the screen as, for example, HAXOR, NO YES YES. (Note

\footnotetext{
${ }^{1}$ The success of a cue is defined as: $d \times v+(1-d) \times 0.5$, where $d$ is the discrimination rate of the cue and $v$ is the cue validity. $d \times v$ is the expected proportion of correct inferences from occasions when the cue discriminates. $(1-d) \times 0.5$ is the expected proportion of correct inferences from occasions when the cue does not discriminate, forcing a guess (with a .50 probability of a correct choice in a two-alternative forced-choice task).
} 


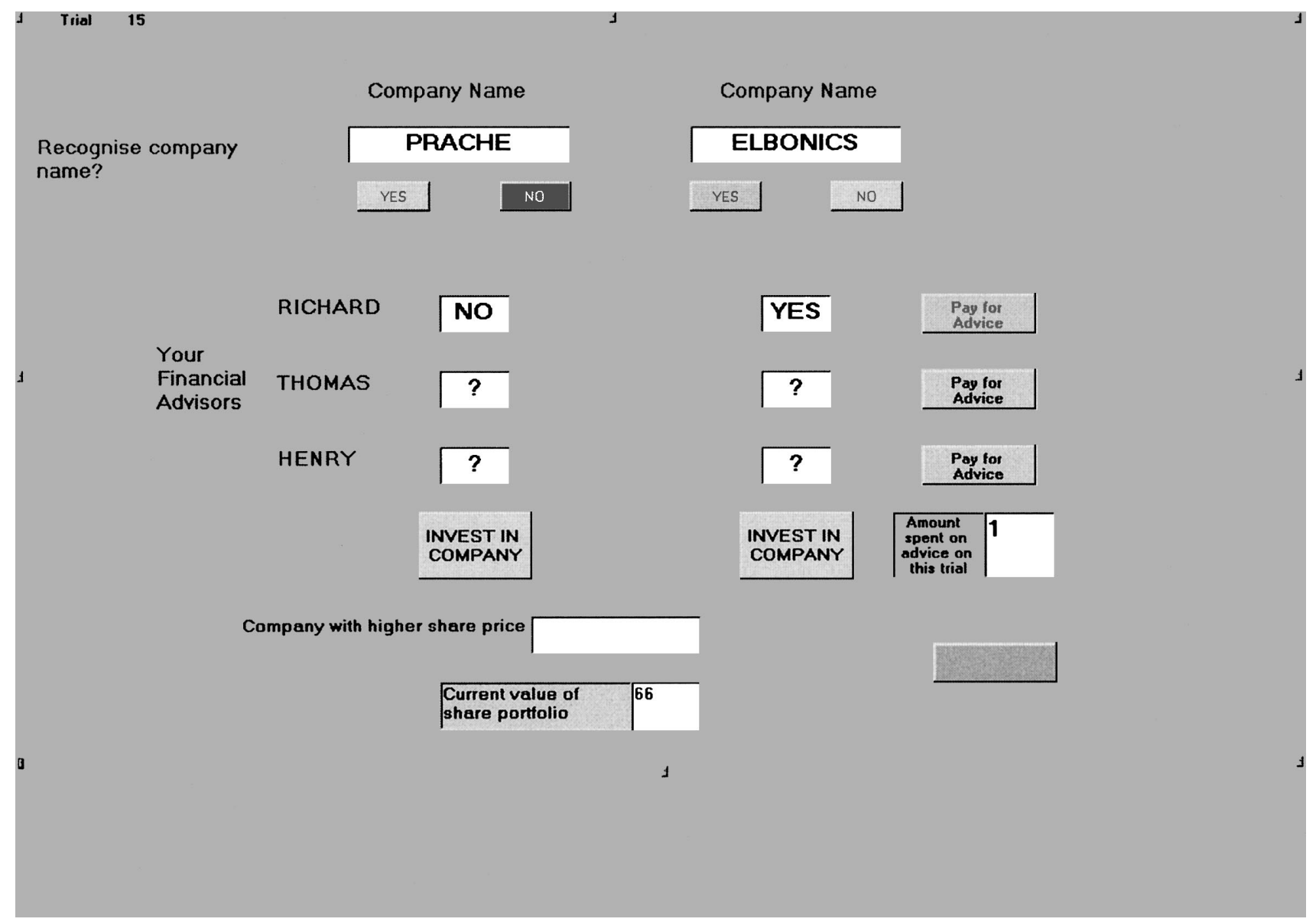

Figure 1. Screenshot of Experiment 1.

that the NOs and YESes were revealed only if participants bought advice). The 16 patterns resulted in 120 possible paired comparisons; however, we only required comparisons in which one cue always discriminated, that is, in which it had a discrimination rate of 1.0. In each condition, the most valid cue was given a discrimination rate of 1.0 , whereas the remaining three cues had discrimination rates of .50 . By constraining the set so that one cue always discriminated, ${ }^{2}$ we reduced the number of pairs to 64 . It is important to note that for the $\mathrm{RH}$ group, participants were able to rely solely on recognition on every trial (if they chose to) once they had learned the names of the repeated companies, because the discrimination rate of 1.0 meant that on each trial, one of the four repeated company names was always paired with a novel company name. In contrast, for the RL group, the discrimination rate of .50 meant that on half of the trials, one of the four repeated names was paired with another of the repeated names, and on the other half, it was paired with a novel name, reducing the number of trials on which they could rely solely on recognition to 32 . Thus, to increase the number of data points and the reliability of the results, we showed the 64 comparisons to all groups twice for a total of 128 trials.

For each comparison, there was an associated probability of one company being more profitable. After each choice, the posterior probabilities that the chosen company was more profitable were computed according to Bayes's rule, assuming conditional independence of the cues. (In their appendix, Newell \& Shanks, 2003, provide details of the calculation method.) Using a random-number generator, we then determined which company was more profitable according to this probability.

Procedure. Participants were first told that they would be taking part in a stock market game. Detailed written instructions on the screen ex- plained that on a series of trials they would be required to choose to invest in one of two companies. Participants in the two recognition conditions were told that their first task was to decide whether they recognized a company. Care was taken to explain that recognition of a company was restricted to the context of the experiment. Participants were told that all of the company names were fictional, so any similarities to real companies should be disregarded. It was explained that throughout the experiment, some company names would be repeated, and so participants should start to gradually recognize the repeated names. Once participants had indicated (via a button press) whether they recognized the companies, the remaining buttons on the screen were enabled. In the NR condition, the free advice from the advisor John was displayed on the screen at the start of each trial. Figure 1 shows a screenshot from the experiment.

At this point, participants could either make their investment decision (purely on the basis of the company name or the free advice) or acquire information from the financial advisors by clicking on a screen button that revealed the direction of the advice (YES or NO) for each company. Information cost $1 \mathrm{p}(100 \mathrm{p}=\mathrm{UK} £ 1=\mathrm{U} . \mathrm{S} . \$ 1.57=$ Euro1.57), one tenth of the potential gain for a correct investment decision (10 p). Participants bought as much or as little information as they desired and then selected the company in which they wished to invest. They were then told which

${ }^{2}$ Constraining the set of comparisons in this way slightly affected the validities assigned to each cue. The resulting experienced validities in both experiments were on average approximately $.05(S D=0.02)$ lower than the programmed validities. 
company had been the correct investment, and if they had made the correct choice, the value of their portfolio was incremented by $10 \mathrm{p}$ minus any money they had spent on buying advice.

Participants were told that not all the advisors were equally good, and that therefore it would be worthwhile to try to work out which ones gave the better advice. In addition, they were told that because our stock market fluctuated, and because the advisors were monitoring these fluctuations, participants should not assume that because an advisor recommended investing in a particular company on one trial that they would always recommend investing in that company. This clarification was necessary because the nature of the design led to the same advisor sometimes recommending investment in one of the repeated companies and sometimes not recommending investment in it.

On completing 128 trials, participants were asked to rate each cue (company name or free advice, and the advice of Tom, Richard, and Henry) on a scale from 0 to 100 where 0 indicated not at all useful and 100 indicated as useful as a piece of information could be for this type of task. After providing the ratings, participants were debriefed and paid.

\section{Ecological Analysis of the Experimental Environment}

It is informative, before examining the results, to consider the performance of strategies that rely to a greater or lesser extent on recognition-based information. To illustrate, we consider two possible strategies participants could adopt in the two conditions. Recall that the reward for a correct decision is $10 \mathrm{p}$ and each piece of advice costs $1 \mathrm{p}$, but the company-name information is free.

In the RH condition, making choices purely on the basis of recognition would provide an expected payoff $(E)$ of

$$
E(\text { Recognition } / \mathrm{RH})=.80 \times 1.0 \times 10 \mathrm{p}=8 \mathrm{p},
$$

where .80 is the validity, 1.0 is the discrimination rate of the company-name cue, and $10 \mathrm{p}$ is the reward on each trial. In contrast, if we were using a strategy in which the advice of the most valid advisor was always bought, the expected payoff would be

$E($ Recognition + Advisor 1/RH)

$$
=(.80 \times 1.0 \times 10 \mathrm{p})-(1 \mathrm{p})=7 \mathrm{p} .
$$

The first term in Equation 2 refers to the expected payoff from relying on recognition, and the second term refers to the cost of the information from the most valid advisor. Note that with such a strategy, accuracy remains the same, because the advice of the most valid advisor has a lower validity than the company-name information and can therefore never compensate for or overrule the information provided by the company-name cue. However, the advice costs $1 \mathrm{p}$, so the expected payoff is necessarily lower.

In the RL condition, the situation is reversed. Now because the company-name cue has a validity of .65 and a discrimination rate of .50 , the expected payoff for relying solely on recognition is

$$
\begin{aligned}
& E(\text { Recognition/RL }) \\
& \quad=(.65 \times .50 \times 10 \mathrm{p})+(.50 \times .50 \times 10 \mathrm{p})=5.75 \mathrm{p} .
\end{aligned}
$$

Here the first term is the expected payoff when the companyname cue discriminated, and the second term is the expected payoff from guessing (i.e., when the company-name cue did not discriminate). However, in the RL condition, the expected payoff when the advice of the most valid advisor was always bought (regardless of whether the company-name cue discriminated) is
$E($ Advisor $1+$ Recognition/RL)

$$
=(.80 \times 1.0 \times 10 \mathrm{p})-(1 \mathrm{p})=7 \mathrm{p} .
$$

This is the same amount as that shown in Equation 2, but now both terms refer to Advisor 1, whose advice had a validity of .80 and discrimination rate of 1.0 (first term), but who charged $1 \mathrm{p}$ for the advice (second term). Even with the cost of the advice, the expected payoff is still greater than it is in Equation 3, because the advice of the most valid advisor is more valid than the recognition information.

This ecological analysis demonstrates that in the RH condition, there is no benefit (at least in terms of earning money) in buying advice, whereas in the RL condition, the best strategy is to buy advice and then make a choice. Thus, to the extent that participants are motivated purely by financial gain, it is beneficial for them to adopt a "pure" recognition-based strategy in the RH condition but (contrary to the recognition heuristic) to buy and rely on the more valid advice in the RL condition. (Note that for the NR condition, the expected payoffs are identical to those in the RH condition, because only the labeling of the most valid cue-free advice or company name, respectively-differs between these conditions.)

\section{Results}

A significance level of .05 was set for all of the statistical tests reported, unless otherwise stated.

Proportion correct. The proportions of trials on which participants made the "correct" investment (i.e., invested in the company that ended up with the higher share price) were $.69, .67$, and .73 for the RH, RL, and NR conditions, respectively. There was no significant effect of condition on proportion correct, $F(2,35)=$ $2.53, \eta^{2}=0.13$. All proportions were significantly above chance performance $(.50), t \mathrm{~s}(11)=9.65,7.16$, and 18.98 for the $\mathrm{RH}, \mathrm{RL}$, and NR conditions, respectively.

Earnings. To ensure that participants had adequate experience in exploring the experimental environment, and to allow for some stability to develop in adopted strategies, we restricted our analysis to the second block of 64 trials. In this block, participants earned $£ 4.18$, £3.89, and $£ 4.32$ in the RH, RL, and NR conditions, respectively. There was no significant effect of condition on amount earned, $F(2,35)=1.17, \eta^{2}=0.07$. In each case, the amount earned was less than the expected earnings from adopting either of the strategies described in the ecological analysis. For RH and NR, relying on the most valid piece of information would have earned $8 \mathrm{p} \times 64=£ 5.12$ (see Equation 1); for RL, the amount would be $7 \mathrm{p} \times 64=£ 4.48$ (see Equation 4). Overall, earnings reflected individual variability in the strategies adopted (see the Analysis of individual data section) and some tendency toward overpurchase of advice, suggesting that financial gain was not the sole motivator for all participants. It is quite possible that nonfinancial considerations influenced their behavior (and perhaps differentially for participants). For example, if participants valued correct responses over and above earnings, or perceived their task, in part, as gaining knowledge or reducing uncertainty (see Lindley, 1956; Oaksford \& Chater, 1998), then it is likely that some overpurchase would occur.

Proportion of trials on which only one company was recognized. Consistent with the analysis of earnings, for the behavioral measures, we restricted our analysis to the second block of 64 trials. 
The interpretation of the results relies on the success of our manipulation to induce recognition of the four repeated company names. Participants indicated via a button press whether they recognized each company. The values in Table 2 indicate that our manipulation was successful. Participants' recognition accuracy was almost perfect in both conditions.

In addition, all measures for the two recognition conditions were conditionalized on whether participants believed that the company-name cue discriminated, and therefore were able to rely solely on recognition information.

Proportion of trials on which the recognized company was chosen. As shown in the predictions in Table 1, we suggested that if recognition is special, participants should choose the recognized company on all the trials on which the company-name cue discriminated.

As we indicate in Table 2, the RH environment participants chose the recognized company on almost $90 \%$ of the trials. However, for the RL environment, there was no suggestion that recognition was treated with an elevated status. Participants in the RL environment chose the recognized company on only $62 \%$ of trials on which they recognized one company and not the other. The difference between these proportions was highly significant, $F(1$, $23)=21.56, \eta^{2}=0.49$. In this environment, such a choice was appropriate because the company-name cue had a validity of only .65. It appears that participants learned that recognizing a company name was not the best predictor of future company profits and thus treated it in the same way as they would any other low-validity cue. The results for the NR condition suggest that the free advice was treated in much the same way as the company-name informa-

Table 2

Group Data for the Second Block of 64 Trials in Experiments 1 and 2

\begin{tabular}{|c|c|c|c|c|}
\hline $\begin{array}{l}\text { Experiment } \\
\text { and condition }\end{array}$ & $\begin{array}{l}\text { Proportion } \\
\text { of correct } \\
\text { recognition } \\
\text { responses }\end{array}$ & $\begin{array}{c}\text { Advice } \\
\text { purchased }\end{array}$ & $\begin{array}{c}\text { Recognized } \\
\text { company } \\
\text { chosen }\end{array}$ & $\begin{array}{c}\text { Contradictory } \\
\text { advice }\end{array}$ \\
\hline $1 \mathrm{RH}$ & 1.00 & .32 & .88 & .34 \\
\hline $1 \mathrm{RL}$ & .94 & .64 & .62 & .84 \\
\hline $1 \mathrm{NR}$ & & .40 & .92 & .15 \\
\hline 2 & .94 & .48 & .66 & .57 \\
\hline
\end{tabular}

Note. There are no data for the NR condition in the proportion column because there were no company names in this condition. For the RH and RL conditions and Experiment 2, all measures are conditionalized on whether participants believed the company-name cue discriminated. Proportion of correct recognition responses refers to trials on which participants indicated that they recognized one company and not the other and were correct (i.e., the trial consisted of a novel company name paired with a repeated name) divided by those on which they were incorrect (i.e., the trial consisted of two novel names or two repeated names). Advice purchased refers to the proportion of trials on which only one company was recognized but on which participants went on to purchase advice. Recognized company chosen refers to the proportion of trials on which participants chose the company they recognized (RH and RL condition and Experiment 2) or the one advised by the free advice (NR condition). Contradictory advice is a proportion derived from dividing the number of trials on which participants purchased advice that either contradicted or created a tie with recognition, and led to the choice of the unrecognized company, by the number of trials on which such contradictory advice was bought. $\mathrm{RH}=$ recognition high; $\mathrm{RL}=$ recognition low; $\mathrm{NR}=$ no recognition. tion in the RH condition (with which it shared the same informational properties). Participants chose the company pointed to by the free advice on the vast majority of trials. There was no significant difference between the proportion of trials on which the most valid cue (company name or free advice) was followed in the two conditions, $F(1,23)=1.47, \eta^{2}=0.06$.

Proportion of trials on which advice was purchased. If recognition alone is sufficient for participants' choices in this task, we would expect no, or very little, advice to be purchased in the RH or RL conditions when the company-name cue discriminated. In addition, we might expect greater advice purchase in the NR condition. In contrast, if recognition is treated in the same way as other cues, then we might expect to see higher purchase of advice in the RL condition than in the RH condition and a similar low level of purchase in the RH and NR conditions (see Table 1).

As Table 2 indicates, this latter pattern is what we found. Participants in the RL condition purchased advice on a significantly higher proportion of trials than did participants in the $\mathrm{RH}$ condition, $F(1,23)=5.76, \eta^{2}=0.21$. There was no significant difference between the proportion of trials on which advice was purchased in the RH and NR conditions, $F(1,23)=.25, \eta^{2}=$ 0.01. Finally, even though the numerical difference in the proportion of trials on which advice was purchased in the NR and RL conditions was large, it did not reach statistical significance, $F(1$, 23) $=2.70, \eta^{2}=0.11$.

It is interesting to note that even the participants in the $\mathrm{RH}$ and NR conditions bought advice on an average of over a third of the trials. This amount seems quite high, given that the company-name or free-advice information was highly valid, discriminating, and free. There was, however, a degree of individual variability in the advice purchase behavior in all conditions as discussed in the Analysis of individual data section. This variability is consistent with patterns of information acquisition reported previously in similar tasks (Bröder, 2000; Newell \& Shanks, 2003; Newell et al., 2003).

Purchase of contradictory advice led to unrecognized company chosen. Our most crucial measure for examining the way in which recognition information was used is the degree to which the purchase of advice that pointed in the opposite direction to the company-name cue led to choosing the unrecognized company. We examined all those trials on which participants bought advice that contradicted the information provided by the company name or free advice or on which the advice bought created a tie between the two companies (e.g., Advisor 1 recommended buying the recognized company and Advisor 2 the unrecognized one). We then calculated the proportion of these trials on which participants chose the unrecognized company. We present the results in the rightmost column of Table 2. Clearly, this behavior was present in both the RH and, especially, RL conditions (contrary to the prediction that recognition information has a special status). The proportion of trials on which this behavior was observed was significantly higher in the RL condition than it was in the $\mathrm{RH}$ condition, $F(1,23)=25.96, \eta^{2}=0.54$.

It is important to note that the inverse of the values for RL and $\mathrm{RH}$ in the rightmost column of Table 2 gives an indication of the reliance on recognition in the presence of contradictory advice. Thus it can be seen that in the RL condition recognition is effectively ignored on all but $16 \%(1-.84=.16)$ of trials. This result provides strong evidence that participants learned to discount 
recognition-based information in the presence of contrary information from a more valid source.

The difference between the $\mathrm{RH}$ and the NR conditions did not reach significance, $F(1,23)=3.42, \eta^{2}=0.13$, again suggesting that in these two informationally equivalent conditions, behavior was largely similar and unaffected by the labeling of the cue as company name or free advice. It is interesting, though, that the trend is in the direction opposite that predicted by a recognitionspecial hypothesis. If anything, free advice was less likely to be overridden by contradictory advice than was recognizing a company name.

Estimated usefulness of cues. On completing 128 trials, participants provided a rating of how useful they thought each cue had been in making their decisions. Recall that the assignment of validities to the advisors was counterbalanced across participants. In Table 3, we display the mean normalized estimated usefulness scores for each cue.

The usefulness ratings indicate that company name was rated as being more useful than the advice from any of the three advisors in the RH condition. Paired-sample $t$ tests confirmed this pattern, $t \mathrm{~s}(11)=3.86,2.70$, and 5.06 for company name versus Advisors 1,2 , and 3 , respectively. The relative similarity of the ratings for the three advisors reflects the fact that the majority of participants bought little advice and thus did not have many opportunities to learn about the relative usefulness of the three advisors. The slightly elevated rating for the second most valid advisor appears to be an artifact of the counterbalancing conditions. Two participants in Condition C (Participants 9 and 6) bought a large amount of advice from the advisor positioned directly under the companyname information (i.e., the first advisor in the list). In this counterbalanced condition, this advisor happened to be the second most valid. These participants therefore rated the advisor as useful, presumably because his was the only advice that they bought.

In the RL condition, participants learned that the advice given by the most valid advisor was the most useful piece of information for making decisions. Paired-sample $t$ tests indicated that this pattern was statistically reliable, $t \mathrm{~s}(11)=2.11(p<.06), 2.85$, and 3.81, for Advisor 1 versus company name, Advisor 2, and Advisor 3 , respectively. However, recognition was rated as equally useful to the advice of the second most and least valid advisor, despite actually having lower predictive validity $(p s>.05)$. This result may be due to participants' having had more chance to sample the company-name cue because it was free. Alternatively, it could suggest that, at least in terms of usefulness, there is a bias toward recognition having an elevated status.

Table 3

Mean Normalized Estimated Usefulness Values for the Four Cues in Experiments 1 and 2

\begin{tabular}{ccccc}
\hline $\begin{array}{c}\text { Experiment and } \\
\text { condition }\end{array}$ & $\begin{array}{c}\text { Company } \\
\text { name/free advice }\end{array}$ & Advisor 1 & Advisor 2 & Advisor 3 \\
\hline $1 \mathrm{RH}$ & .48 & .17 & .24 & .11 \\
$1 \mathrm{RL}$ & .26 & .37 & .20 & .16 \\
$1 \mathrm{NR}$ & .47 & .16 & .18 & .19 \\
2 & .29 & .30 & .22 & .18 \\
\hline
\end{tabular}

Note. $\mathrm{RH}=$ recognition high; $\mathrm{RL}=$ recognition low; $\mathrm{NR}=$ no recognition.
The pattern for the NR condition matched that of its informational equivalent, the RH condition. The free advice was rated as more useful than the advice of the other three advisors, $t \mathrm{~s}(11)=$ 4.02, 3.86, and 4.57 for free advice versus Advisors 1, 2, and 3, respectively. There was also no difference between the rating given for the company-name cue in the $\mathrm{RH}$ condition and the free-advice cue in the NR condition, $t(22)=0.08$, providing evidence contrary to the suggestion that recognition information is attributed special status in this task.

Analysis of individual data. Table 4 presents individual participant data for the three conditions of Experiment 1. Presenting the data in this way indicates that although the majority of participants in each condition behaved in a manner consistent with the pattern borne out by the group analysis, there were some notable exceptions.

In the RH condition, Participants 9 and 10 (and to a lesser extent, Participant 6) appear not to be relying solely on recognition information. All three bought advice on the majority of trials and, more importantly, often followed that advice when it pointed in the direction opposite to that of the company-name cue.

In the RL condition, Participants 15, 16, and 20 still appear to rely largely on recognition despite the low validity of the company-name cue. These participants rarely bought advice (.17 of trials on average), but, interestingly, when the advice was contradictory, they very often followed it (.82 of trials on average).

Perhaps the most extreme individual variations are in the NR condition. Four participants (Participants 27, 28, 35, and 36) bought advice on the vast majority of trials (over .70), in contrast to the other 8 participants in the group, most of whom bought advice on less than .10. Furthermore, the contradictory advice bought by Participants 27, 28, 35, and 36 appeared to increase the proportion of trials on which they chose the alternative not pointed to by the free advice (an average of .27 compared with .09 for the remaining 8 participants).

\section{Discussion}

We designed Experiment 1 to examine the use of recognitionbased information in a cue-learning task and to test the plausibility of assigning recognition information a special status in decision making. We found clear evidence that participants were sensitive to the validity of recognition and that the majority used the information appropriately. When recognition information was highly valid, participants used the information, often without buying any other information. However, when recognition information was no longer the best predictor of company success, participants did not base their decisions solely on recognition but chose to buy advice from at least one advisor (typically the one with the highest validity). There was no suggestion that recognition was treated in a special way or that it biased participants to make inappropriate decisions.

In one sense, this pattern of results fits very well with the adaptive toolbox approach, in that participants learned to use the information provided by recognition in appropriate ways in the different experimental conditions. What the results do not support, however, is the claim that people treat recognition information in a way that is qualitatively different from the way they treat other information in the environment. Juslin and Persson (2002), in discussing the way in which their PRoBEX model makes infer- 
Table 4

Individual Data for Behavioral Measures in Experiment 1

\begin{tabular}{|c|c|c|c|c|}
\hline \multirow[b]{2}{*}{ Participant } & \multirow[b]{2}{*}{ Condition } & \multicolumn{3}{|c|}{ Proportion of final 64 trials } \\
\hline & & $\begin{array}{c}\text { Advice } \\
\text { purchased }\end{array}$ & $\begin{array}{c}\text { Recognized } \\
\text { company chosen }\end{array}$ & $\begin{array}{c}\text { Contradictory } \\
\text { advice }\end{array}$ \\
\hline 1 & $\mathrm{RH}$ & .02 & 1.00 & .00 \\
\hline 2 & RH & .00 & .83 & .00 \\
\hline 3 & $\mathrm{RH}$ & .08 & .97 & .66 \\
\hline 4 & $\mathrm{RH}$ & .08 & .98 & .00 \\
\hline 5 & $\mathrm{RH}$ & .14 & .98 & .00 \\
\hline 6 & RH & .72 & .86 & .29 \\
\hline 7 & RH & .09 & .94 & .80 \\
\hline 8 & $\mathrm{RH}$ & .17 & .84 & .80 \\
\hline 9 & $\mathrm{RH}$ & .88 & .73 & .32 \\
\hline 10 & RH & .98 & .72 & .43 \\
\hline 11 & $\mathrm{RH}$ & .41 & .89 & .35 \\
\hline 12 & $\mathrm{RH}$ & .23 & .80 & .45 \\
\hline 13 & RL & .70 & .60 & .67 \\
\hline 14 & RL & .71 & .56 & 1.00 \\
\hline 15 & RL & .21 & .85 & .71 \\
\hline 16 & RL & .18 & .64 & .75 \\
\hline 17 & RL & .60 & .80 & .67 \\
\hline 18 & RL & 1.00 & .42 & .94 \\
\hline 19 & RL & 1.00 & .44 & 1.00 \\
\hline 20 & RL & .13 & .90 & 1.00 \\
\hline 21 & $\mathrm{RL}$ & 1.00 & .43 & 1.00 \\
\hline 22 & RL & .72 & .69 & .75 \\
\hline 23 & RL & .84 & .44 & .74 \\
\hline 24 & RL & .63 & .63 & .80 \\
\hline 25 & NR & .45 & .97 & .11 \\
\hline 26 & NR & .34 & .97 & .15 \\
\hline 27 & NR & 1.00 & .88 & .18 \\
\hline 28 & NR & .98 & .92 & .13 \\
\hline 29 & NR & .06 & .97 & .00 \\
\hline 30 & NR & .06 & .97 & .50 \\
\hline 31 & NR & .02 & 1.00 & .00 \\
\hline 32 & NR & .02 & .89 & .00 \\
\hline 33 & NR & .09 & 1.00 & .00 \\
\hline 34 & NR & .02 & 1.00 & .00 \\
\hline 35 & NR & .70 & .77 & .37 \\
\hline 36 & NR & 1.00 & .75 & .41 \\
\hline
\end{tabular}

Note. For the RH and RL conditions, all measures conditionalized on whether participants believed that the company-name cue discriminated. Advice purchased refers to the proportion of trials on which only one company was recognized but on which participants bought advice. Recognized company chosen refers to the proportion of trials on which participants chose the company they recognized (RH and RL condition) or the one advised by the free advice (NR condition). Contradictory advice is a proportion derived from dividing the number of trials on which participants purchased advice that either contradicted or created a tie with recognition, and led to the choice of the unrecognized company, by the number of trials on which such contradictory advice was bought. For example, Participant 9 purchased advice on .88 of trials on which he recognized only one company, chose the recognized company on .73 of trials, and chose the unrecognized company on .32 of the trials on which contradictory advice was bought. $\mathrm{RH}=$ recognition high; $\mathrm{RL}=$ recognition low; $\mathrm{NR}=$ no recognition.

ences in the German cities task, drew a similar conclusion with regard to recognition, stating, "recognition is a 'probability cue' among others" (p. 37).

A key difference, however, between the environment used in Experiment 1 and the types of environments in which recognition might be attributed an elevated status is that in Experiment 1, participants were able to buy advice about unrecognized compa- nies. In the domains for which the recognition heuristic was formulated (e.g., the German cities environment), it is impossible to search one's memory for information about unrecognized objects (i.e., if you don't recognize a city, you cannot search your memory to discover whether the city has a soccer team). Despite this, the impossibility of further search seems rather unrepresentative of many everyday decisions that involve recognition. For example, when buying a new computer, a person might recognize one brand over another, but rather than simply purchasing the recognized brand, she might find further information about the unrecognized brand (by looking in a magazine, for example). Perhaps this opportunity for further search about unrecognized alternatives is a critical mediating variable for the use of recognition in decision making.

A participant in Experiment 1 might reason that because the advisors had taken the trouble to find out about a particular unrecognized company, their advice might well be worth obtaining. Such an interpretation would influence the importance placed on the recognition information. Furthermore, the strong claim expounded in the quote from Goldstein and Gigerenzer (2002) in the introduction to this article concerns the curtailment of search for information about the recognized alternative (once it has been recognized) and makes no mention of the unrecognized alternative. In Experiment 2, we attempted to address these concerns by modifying the experimental design such that now advice could only be bought about recognized companies.

\section{Experiment 2}

We designed Experiment 2 to test the notion that once an alternative has been recognized, it will be chosen regardless of the opportunity to obtain further information about that alternative and regardless of what the information might indicate about the suitability of that alternative. The key point of interest in the context of the current task is whether advice that recommends against investment in a recognized company leads to the choice of the unrecognized company. Given the reliance on recognition we observed in the RH condition of Experiment 1, it seemed unnecessary to include this condition in Experiment 2. The condition of concern is RL, in which other cues in the environment have a higher predictive validity than recognition and therefore can be used to make more accurate decisions. The question is: Will participants learn to use these other cues, or will the special status of recognition override the temptation to search for and use extra information?

\section{Method}

Participants. Twelve members ( 7 women and 5 men, mean age $=20.6$ years) of the University College London community participated in the experiment in return for performance-related remuneration.

Stimuli and design. The design was closely related to the RL condition of Experiment 1 with the crucial difference that now when a company was unrecognized, no advice relating to that company could be obtained. (The program was modified so that the Buy Advice buttons were disabled when a company was not recognized.) The validities assigned to each of the cues were .60 for the company-name cue and $.85, .75$, and .65 for the three advisors. The validities assigned to the advisors were raised slightly to increase the likelihood that participants would learn about the usefulness of the advice during the experiment, even when they had fewer opportunities 
to sample the advice (opportunities were fewer because of the restriction that advice could only be bought about recognized companies). The validity of recognition was lowered slightly, again to reflect the imbalance in exposure to the sources of cue information (recall that the company-name cue was free on every trial). Discrimination rates were held constant with 1.0 for the most valid advisor and .50 for company name and the remaining advisors. All participants completed 128 trials and then made usefulness ratings for the four cues.

Procedure. The instructions were identical to those used in Experiment 1 with the exception of the following paragraph, inserted to explain why advice could only be bought about recognized companies: "Your advisors have similar experience to you and so if you don't recognize a company, then neither will they, and in such circumstances they won't be able to offer you advice". Consistent with Experiment 1, participants were encouraged to be as accurate as possible in their recognition responses. Accuracy was emphasized to reduce any temptation to give incorrect recognition responses and thus falsely acquire advice about companies (behavior that, in fact, was not observed - see Recognition accuracy section).

\section{Results}

Proportion correct. The proportion of trials on which participants made the correct investment was .58 and was significantly above chance (.50), $t(11)=5.00$. The slight reduction in the proportion correct compared with that achieved in Experiment 1 $(M=.69)$ is a reflection of the fact that in this experiment, participants were forced to guess on $25 \%$ of the trials (those in which neither company was recognized). Assuming that participants were incorrect on approximately half of these trials would allow one to account for the difference in overall proportion correct achieved in the two experiments.

Earnings. Participants earned an average of $£ 3.53$ in the second block of 64 trials. This slightly lower total than that earned by the equivalent group in Experiment 1 (RL, $M=£ 3.89$ ) again reflects the fact that participants were forced to guess on $25 \%$ of the trials, hence lowering earnings.

Recognition accuracy. We display recognition accuracy for the second block of 64 trials in the fourth row of Table 2, which shows that accuracy was identical with that in the RL condition of Experiment 1 and highlights that participants did not make false claims about recognizing companies in order to obtain advice. Consistent with Experiment 1, all behavioral measures were restricted to an analysis of this second block of 64 trials and were conditionalized on whether participants believed that the company name cue discriminated and were therefore able to rely solely on recognition information.

Proportion of trials on which recognized company was chosen. We display the proportion of trials on which the recognized company was chosen in Table 2 . The proportion is highly consistent with that found in the RL condition of Experiment 1. Indeed, a cross-experiment comparison revealed that there was no significant difference between these two proportions, $F(1,23)=.228$, $\eta^{2}=0.01$. The absence of a difference suggests that preventing participants from accessing information about unrecognized companies had little impact on the degree to which recognition-based information influenced decision behavior.

Proportion of trials on which advice was purchased. In the fourth row of Table 2, we display the proportion of trials on which participants bought advice when they believed the company-name cue discriminated. This advice, necessarily, was advice only about the recognized company. Although it was numerically smaller than the value found in the RL condition of Experiment 1, the difference was not statistically reliable, $F(1,23)=1.12, \eta^{2}=0.05$, reflecting the large degree of individual differences in the measures (see Tables 4 and 5). Again, the implication of this result is that at a group level, the absence of advice about unrecognized alternatives did not significantly affect decision behavior.

Purchase of conflicting advice led to unrecognized company chosen. When participants bought advice about the recognized company that recommended against investment, how often did they then invest in the unrecognized company? At a group level, the mean proportion of trials on which this behavior was observed was .57. Again, although numerically lower than that observed in the RL condition of Experiment $1(.84)$, the difference was not statistically reliable, $F(1,23)=3.93, \eta^{2}=0.15$, once again reflecting individual variability in the measures-see Analysis of individual data section.

To gain further insight into the effect of advice on participants' decisions, we compared the proportion of trials on which the recognized company was chosen when advice conflicted with recognition with the proportion on which the recognized company was chosen when advice was consistent with recognition. Note that if further knowledge about recognized alternatives is irrelevant when recognition can be relied on (Goldstein \& Gigerenzer, 2002), then we should expect no difference between these two proportions. The recognized alternative should be chosen regardless of the advice. In stark contrast to this prediction, when advice conflicted, the proportion of trials on which the recognized company was chosen was .13 , whereas when advice was consistent with recognition, the proportion was .93 ; this was a highly significant difference, $t(8)=11.59 .^{3}$

Taken together, the overwhelming pattern from the group data is that the crucial manipulation in Experiment 2-removing the opportunity to obtain information about unrecognized alternatives - did not have a significant impact on the use of cue information in the environment and the subsequent decisions that were made.

Estimated usefulness of cues. In the fourth row of Table 3, we display the mean normalized estimated usefulness scores for each cue. The ratings indicate that, consistent with the RL condition of Experiment 1, the advice given by the most valid advisor was rated as the most useful piece of information for making the investment decisions. Paired sample $t$ tests indicated that this pattern was statistically reliable for Advisor 1 versus Advisor 2 and for Advisor 1 versus Advisor 3, $t(11)=2.20, p=.05$, and $t(11)=2.30$, respectively. However, the difference between the usefulness rating of Advisor 1 and the company-name cue was not reliable, and company name was rated as significantly more useful than the advice of Advisors 2 and 3, despite having a lower validity. This latter result is consistent with the RL condition of Experiment 1 and provides some support for recognition of company name being perceived as more than just another cue.

\footnotetext{
${ }^{3}$ This analysis is conditionalized on whether any advice was bought at all (i.e., by excluding Participants 2, 8, and 12-see Table 5). If these participants are included, the proportions become .10 and .70 for conflicting and consistent advice, respectively, and the difference is still highly significant, $t(11)=5.16, p<.01$.
} 
Table 5

Individual Data for Behavioral Measures in Experiment 2

\begin{tabular}{cccc}
\hline & \multicolumn{3}{c}{ Proportion of final 64 trials } \\
\cline { 2 - 4 } Participant & $\begin{array}{c}\text { Advice } \\
\text { purchased }\end{array}$ & $\begin{array}{c}\text { Recognized } \\
\text { company chosen }\end{array}$ & $\begin{array}{c}\text { Contradictory } \\
\text { advice }\end{array}$ \\
\hline 1 & .21 & .89 & 1.00 \\
2 & .00 & 1.00 & .00 \\
3 & .65 & .61 & 1.00 \\
4 & 1.00 & .44 & .95 \\
5 & .35 & .90 & .60 \\
6 & 1.00 & .58 & .92 \\
7 & 1.00 & .53 & .79 \\
8 & .00 & .26 & .00 \\
9 & .50 & .62 & .57 \\
10 & 1.00 & .50 & .00 \\
11 & .03 & .97 & .00 \\
12 & .00 & .61 & .00 \\
\hline
\end{tabular}

Note. Advice purchased refers to advice bought about the recognized company on trials on which only one company was recognized. Contradictory advice is a proportion derived from dividing the number of trials on which participants purchased advice that either contradicted or created a tie with recognition, and led to the choice of the unrecognized company, by the number of trials on which such contradictory advice was bought.

Analysis of individual data. In Table 5, we display the individual data for the behavioral measures described above. The clear majority of participants (Participants 1, 3, 4, 5, 6, 7, 9, and $10-75 \%$ ) followed the patterns borne out by the group analysis. These participants bought advice on a significant proportion of trials (min. $=.21, \max .=1.00, M=.71$ ) and chose the company pointed to by advice when it conflicted with the company-name information $(\min .=.57, \max .=1.00, M=.85)$. A minority of participants (Participants 2, 8, 11, and 12-25\%) behaved in a manner more consistent with the notion that recognition of company name exerted a strong influence on their investment decisions. Notably, Participants 2 and 11 almost never bought advice when they believed the company-name cue discriminated, and nearly always invested in the company that they recognized. In contrast, Participant 8 appeared to use an antirecognition heuristic, choosing to invest in the unrecognized company on almost $75 \%$ of trials despite never having bought any information. Finally, Participant 12 erred on the side of choosing the recognized company but still chose the unrecognized one on .39 of trials, despite having bought no advice.

\section{Discussion}

The clear result from Experiment 2 is that preventing participants from accessing information about unrecognized alternatives does not have a large effect on decision behavior. When recognition information could be relied on solely, the majority of participants (75\%) purchased some advice and followed the advice, rather than basing their decisions on simple recognition. The data suggest that the opportunity to search for information about an unrecognized alternative is not a critical mediating variable for the use of recognition in this cue-learning task. However, one aspect of the data that lends some modest support to the notion that recognition is treated with an elevated status comes from the usefulness ratings. Here participants tended to overestimate the usefulness of recognizing the company name for making accurate decisions.

\section{General Discussion}

Personal experience tells us that although recognizing an object can often be useful in guiding our decisions, we do not rely solely on recognition. Recognizing the name of a horse might be a good cue for placing a bet, but would we stake a million dollars? This example might seem trivial, but it serves to illustrate the distinction between the claim that recognition often serves as a useful shortcut to good decisions and the notion that recognition has a fundamental, noncompensatory effect on decision making (e.g., Goldstein \& Gigerenzer, 2002).

Our focus in this article was on an experimental examination of the role of recognition information in a cue-learning task. Our data demonstrate that the majority of participants learned to use recognition-based information when it was a good predictor of performance and to essentially ignore it when it was a poor predictor. Thus, the positive aspect of our data is that participants were not biased by recognition-based cues. Recognition did not jump out and override the presence of other information in the environment, regardless of whether that information was about both alternatives (Experiment 1) or only the recognized alternative (Experiment 2). In summary, we found little evidence suggesting that recognition is treated any differently from other cues in the environment.

One exception to this general pattern of recognition being treated in the same way as other cues was that its usefulness as a cue was overestimated. These ratings data provide important evidence to counter a potential criticism of the findings. One could argue that assigning the company-name cue relatively low validity in the RL condition of Experiment 1 and Experiment 2 prevented participants from learning that the cue was indeed a valid predictor of performance (i.e., that it had a predictive validity above .50). In Experiment 2, the nature of the design led to the company-name cue's having an experienced validity of approximately .55 (see footnote 2), thus making it potentially rather difficult for participants to learn in the first 64 trials that it was a valid predictor. The ratings data, however, counter this suggestion by showing that participants believed company name to be a useful piece of information for helping to make decisions. Despite this belief, the majority were not prepared to base decisions solely on recognition-as evidenced by the behavioral data. ${ }^{4}$

Our finding that participants learned about recognition-based information and relied on it appropriately counters the suggestion that recognition exerts a noncompensatory influence on decision making. Given this contrasting result, it is worth considering (a) the evidence marshaled by Goldstein and Gigerenzer (2002) to support the claim for noncompensatory use and (b) differences in the environments used that might account for the discrepant results

\footnotetext{
${ }^{4}$ It is possible that participants rated company name as useful because they chose systematically against recognition - that is, they used an antirecognition heuristic: For example, "The cue is useful because when I recognize a company, I infer that the unrecognized alternative is the better choice." Though a possibility, analysis of the data indicates that only one participant (Participant 8 in Experiment 2) appeared to use such a strategy.
} 
and provide insights into when, where, and why recognition is used in decision making.

\section{Evidence for Noncompensatory Use of Recognition}

In Goldstein and Gigerenzer's (2002) Experiment 2, participants were presented with a series of pairs of German cities and asked to choose the city they believed to have the larger population. In addition to the city names, participants were taught some extra information about some of the cities in the sample that could, Goldstein and Gigerenzer argued, be incorporated into the decisions. Before beginning the cities task, participants were given a training phase in which they were told that 9 of the 30 largest cities in Germany have soccer teams and that the 9 cities with teams are larger than the 21 without teams in $78 \%$ of all possible pairs. They were also taught the names of 4 well-known cities that have soccer teams and 4 that do not. Participants were then tested on this knowledge and were only allowed to continue in the study when they had recalled the information without error.

The critical pairs in the cities task that followed were those that included one unrecognized city and one recognized city that did not have a soccer team. Goldstein and Gigerenzer (2002) argued that, equipped with the knowledge from the training phase and placing no special emphasis on recognition, participants should have chosen the unrecognized city in such pairs. This is because from the information given, participants could work out that if a city does not have a soccer team, then even if it is recognized, it is only likely to be larger than an unrecognized city in $22 \%$ of all possible pairs. Thus any chance that the unrecognized city has a soccer team should lead participants to choose against the prediction of the recognition heuristic.

Despite being provided with this conflicting information, Goldstein and Gigerenzer (2002) reported that participants' inferences followed those of the recognition heuristic on an average of $92 \%$ of the critical pairs. This finding was their key evidence for the noncompensatory use of recognition information. It is clear that the study described provides some evidence of this, but the design that Goldstein and Gigerenzer used was perhaps not ideally suited to test the strong claim about the irrelevance of further knowledge about recognized alternatives. First, the design did not require participants to learn about the validity of information in an incremental fashion - rather, it relied on participants integrating information about percentages provided at training into their test decisions. Second, and more important, because the study did not include a critical control group (i.e., a group whose members were not taught the soccer team information), it is not possible to conclude whether the soccer team information had any effect on performance. Maybe, without that information, participants would have achieved greater than $92 \%$ adherence to the heuristic.

\section{Differences in Environments: Internal Versus External Search}

The pattern of results obtained in both experiments suggests strongly that recognition information is treated differently in the cue-learning task than it is in the German cities environment: Why? An obvious difference is that the former requires participants to search for information both from an external environment (from the advisors on the computer screen), and from memory (in order to infer whether a company name had been repeated), whereas in the latter, the search is only from memory. This difference in environments may have two effects: First, the presence of external attributes (i.e., the advisors) may encourage search and thus reduce reliance on recognition; second, although both tasks involve search from memory, the nature of the inferences made on the basis of this search may be different.

The results of Experiment 2 seem to counter the first argument: Denying participants the opportunity to obtain advice about an unrecognized object did not affect the use of recognition-based cues. Furthermore, it seems fair to equate the financial cost of obtaining advice with the implied cognitive costs of searching one's memory for relevant information in the cities task-thus potentially reducing the apparent differences between the two tasks.

That said, there might still be an aspect of the use of memory in the cue-learning task that changes the influence of recognition in comparison with the cities task. It is possible that in the cities task, when a participant recognizes one city (e.g., Berlin) but not another (e.g., Essen), the participant may not know exactly why one is recognized and the other is not, but may be willing to attribute the feeling of familiarity associated with recognition to the variable in question (e.g., city size). The attribution is presumably made because participants use an intuitive theory that they are more likely to have heard about larger cities (though see Oppenheimer, 2003, for a simple demonstration of when such an effect can be reversed). In contrast, in the cue-learning task, participants presumably have far less uncertainty about the source of the familiarity of repeated nonwords, and therefore have a more clearly specified basis by which to make their attribution.

Perhaps it is the nature of this attribution of familiarity that leads to the reliance on recognition in the cities task. However, such an argument suggests that it is not pure recognition that determines an inference but recognition plus an appropriate reason for knowing why a particular object is recognized-or, at least, a correctly interpreted feeling of familiarity. It is not that an object is recognized and chosen without justification, but that the decision maker has a reasonable idea of why he or she recognizes the object and makes an inference on the basis of this secondary knowledge. Under such an interpretation, it is this secondary knowledge that is precomputed through exposure in an environment (presumably through an associative process) that is the driving force behind the inference, not recognition per se. This explanation is consistent with Kahneman and Frederick's (2002) recent discussion of the recognition heuristic. They propose that one process gives rise to an automatic generation of familiarity when an object is recognized, whereas another, more deliberative, process assesses the basis for the feeling of familiarity (e.g., It is familiar; therefore, it is probably larger; see Stanovich \& West, 2002, for further discussion of dual processes).

In general, such an explanation seems far more consistent with the pattern of results we obtained (although it is not necessary to invoke dual processes to explain the results), and indeed, with the way in which introspection suggests that recognition is used. It also suggests that much of the simplicity of the recognition heuristic is in its description-a description that belies the more complex processes underlying its successful implementation (i.e., knowledge of why recognition is a good predictor in a given environment). This tension between heuristics that can be de- 
scribed in simple terms but that require a good deal of precomputed knowledge to be implemented is a criticism that has been leveled at the adaptive toolbox approach in general (e.g., Chater et al., 2003; Juslin \& Persson, 2002).

Furthermore, explaining the use of recognition in this way seems inconsistent with the bold (and more newsworthy) claims for the existence of a noncompensatory recognition heuristic as a cognitive adaptation that is triggered into action by the properties of particular environments. It is clear that before such claims can be substantiated, we need to have more evidence from different environments that recognition is treated with such an elevated status. The current results do not seem to support such claims; especially those of Experiment 2 in which (a) some but not all alternatives were recognized, (b) recognition validity was greater than chance, and (c) information could only be obtained about recognized alternatives - in other words, a fair experimental analogue of the kinds of situations in which recognition is proposed to exert its noncompensatory influence.

One potentially interesting situation that our experiments did not address is the reliance on recognition when external attributes are readily apparent in the environment. Note that in our task there was an opportunity to obtain information about objects, but this information was not freely presented. This contrasts with many situations that clearly involve recognition in which the external attributes (e.g., designs, color, texture, or even taste of objects) are immediately obvious to the decision maker. An obvious area of research to examine is that of consumer choice. Research shows that companies put a great deal of effort into establishing memorable brand names that will be recognized by consumers (e.g., Aaker, 1991; Alba \& Hutchinson, 1987; Lerman \& Garbarino, 2002), but there is less evidence concerning how brand recognition actually influences choice.

A study by Hoyer and Brown (1990) is an important exception. In that study, participants were faced with a choice among three brands of peanut butter. There were five trials, and after each choice, participants were allowed to taste the brand they had selected. In one group, one brand was known to participants (knowledge of the brand was established through pretest questioning), and in another group, all three brands were unknown. The brand-aware group showed a strong tendency to select the recognized brand on the first trial, and the majority stated that they had explicitly used brand recognition as the basis of their choice. Interestingly, though, as the trials progressed, participants stated that their choices were guided more by the taste of the peanut butter (an external attribute) than by the brand, though the majority still chose the recognized brand. The result suggests that freely presented external attributes do, at least, affect people's interpretation for the basis of their choices, if not, in this case, their actual choices.

\section{On the Adaptivity of Recognition}

Finally, we consider some arguments for the adaptivity of relying on recognition. The question of what the "adaptive" in the "adaptive toolbox" means perhaps needs some clarification (see Over, 2000a, 2000b). As Stanovich and West (in press) recently argued, there is inconsistency as to whether ecological rationality is defined as maximizing for genes (i.e., genetic fitness) or as maximizing for the vehicle (the term used to describe the current agent constructed by the genome- - see Dawkins, 1989). In a textual analysis of Gigerenzer et al. (1999), Stanovich and West (in press) pointed out that the phrase "organism's adaptive goals" is used interchangeably to refer to both the genes' goals and the vehicle's goals. Despite these inconsistencies, Todd, Fiddick, and Krauss (2000), in response to a critique by Over (2000b), claimed that the emphasis of the "adaptive" in the "adaptive toolbox" was on decision making in present environments, without concern for evolutionary fitness. It is beyond the scope of this article to enter the debate about genetic goals versus vehicle's goals, but we can take Todd et al.'s claim at face value and examine how adaptive - in current decision-making environments - it is to rely on simple recognition.

For example, in the peanut-butter study cited earlier, how adaptive was it to rely on brand recognition? It is interesting to note that Hoyer and Brown (1990) found that in a condition in which the higher-quality peanut butter was placed in an unknown brand jar, significantly fewer of the participants in the brand-aware group ended up choosing the higher quality product on the final trial than did participants in the no-awareness group. The interpretation of this effect was that reliance on a brand-recognition heuristic led to reduced search through the alternative unknown brands, thus leading to selection of the inferior product on the final trial. This is a clear example of recognition acting as a bias - a bias on which advertisers regularly capitalize (e.g., "just paying for the name") and a strong indication that we should use other information available in the environment to override simple recognition (cf. Over, 2000b).

Goldstein and Gigerenzer (2002) themselves note that the recognition heuristic can be fooled, but do not seem to extend the logic of their argument to acknowledge that relying solely on recognition seems to have a rather restricted appeal. Another case in point is the degree to which simple reliance on recognition of company names can lead to success on the stock market. Borges, Goldstein, Ortmann, and Gigerenzer (1999) make much of the finding that a portfolio of stocks recognized by over $90 \%$ of Munich pedestrians beat portfolios selected by experts and those of two benchmark mutual funds during the period December 1996June 1997. However, as Boyd (2001) suggested, this effect may simply have been a big-firm effect. High capitalization and high recognition tend to go together (Over, 2000b), and in the strong "bull market" of those months, the high capitalization stocks of the big firms tended to do very well. Boyd emphasized this point by testing the recognition heuristic in a "bear" or down market (JuneDecember 2000). The results were opposite to those found by Borges et al. (1999). Stocks recognized by less than $10 \%$ of the nonexpert participants achieved a return $30 \%$ greater than that achieved by the stocks recognized by more than $90 \%$ and a $20 \%$ higher return than the market index. The message from Boyd's test seems to be that the original finding of Borges et al. was a simple effect of the timing of their study, rather than evidence that recognition per se can beat the stock market.

Thus, relying solely on recognition, whether in the real stock market or in an artificial one (like the one used in the current experiments), is not necessarily the best policy-recognition, like any other indicator of performance, needs to be considered carefully and interpreted, rather than used simply to determine an inference. Importantly, our results suggest that, given the appropriate learning environment, this is indeed what people do. 


\section{References}

Aaker, A. (1991). Managing brand equity. New York: Free Press.

Alba, J. W., \& Hutchinson, J. W. (1987). Dimensions of consumer expertise. The Journal of Consumer Research, 13, 411-454.

Borges, B., Goldstein, D. G., Ortmann, A., \& Gigerenzer, G. (1999). Can ignorance beat the stock market? In G. Gigerenzer, P. M. Todd, \& The ABC Research Group (Eds.), Simple heuristics that make us smart (pp. 59-72). Oxford, England: Oxford University Press.

Boyd, M. (2001). On ignorance, intuition and investing: A bear market test of the recognition heuristic. The Journal of Psychology and Financial Markets, 2, 150-156.

Bröder, A. (2000). Assessing the empirical validity of the "Take-The-Best" heuristic as a model of human probabilistic inference. Journal of Experimental Psychology: Learning, Memory, and Cognition, 26, 13321346.

Bröder, A. (2003). Decision making with the adaptive toolbox: Influence of environmental structure, personality, intelligence, and working memory load. Journal of Experimental Psychology: Learning, Memory, and Cognition, 29, 611-625.

Bröder, A., \& Schiffer, S. (2003). "Take-the-Best" versus simultaneous feature matching: Probabilistic inferences from memory and the effects of representation format. Journal of Experimental Psychology: General, 132, 277-293.

Chater, N., Oaksford, M., Nakisa, R., \& Redington, M. (2003). Fast, frugal and rational: How rational norms explain behavior. Organizational Behavior and Human Decision Processes, 90, 63-80.

Dawkins, R. (1989). The selfish gene (2nd ed.). New York: Oxford University Press.

Dhami, M. K., \& Ayton, P. (2001). Bailing and jailing the fast and frugal way. Journal of Behavioral Decision Making, 14, 141-168.

Elwyn, G., Edwards, A., Eccles, M., \& Rovner, D. (2001). Decision analysis in patient care. The Lancet, 358, 571-574.

Gigerenzer, G. (2001). The adaptive toolbox. In G. Gigerenzer \& R. Selten (Eds.), Bounded rationality: The adaptive toolbox (pp. 37-51). Cambridge, MA: MIT Press.

Gigerenzer, G., \& Goldstein, D. G. (1996). Reasoning the fast and frugal way: Models of bounded rationality. Psychological Review, 103, 650669

Gigerenzer, G., Todd, P. M., \& The ABC Research Group. (1999). Simple heuristics that make us smart. Oxford, England: Oxford University Press.

Goldstein, D. G., \& Gigerenzer, G. (2002). Models of ecological rationality: The recognition heuristic. Psychological Review, 109, 75-90.

Hoyer, W. D., \& Brown, S. P. (1990). Effects of brand awareness on choice for a common, repeat-purchase product. Journal of Consumer Research, 17, 141-148.

Juslin, P., Jones, S., Olsson, H., \& Winman, A. (2003). Cue abstraction and exemplar memory in categorization. Journal of Experimental Psychology: Learning, Memory, and Cognition, 29, 924-941.

Juslin, P., \& Persson, M. (2002). PROBabilities from EXemplars (PROBEX): A "lazy" algorithm for probabilistic inference from generic knowledge. Cognitive Science, 95, 1-4.

Kahneman, D., \& Frederick, S. (2002). Representativeness revisited: Attribute substitution and intuitive judgment. In T. Gilovich, D. Griffin, \&
D. Kahneman (Eds.), Heuristics and biases: The psychology of intuitive judgment (pp. 49-81). Cambridge, England: Cambridge University Press.

Lerman, D., \& Garbarino, E. (2002). Recall and recognition of brand names: A comparison of word and nonword name types. Psychology and Marketing, 19, 621-639.

Lindley, D. V. (1956). On a measure of the information provided by an experiment. Annals of Mathematical Statistics, 27, 986-1005.

Martignon, L., \& Hoffrage, U. (1999). Why does one-reason decision making work? A case study in ecological rationality. In G. Gigerenzer, P. M. Todd, \& The ABC Research Group (Eds.), Simple heuristics that make us smart (pp. 119-140). Oxford, England: Oxford University Press.

Newell, B. R., Rakow, T., Weston, N. J., \& Shanks, D. R. (2004). Search strategies in decision-making: The success of success. Journal of Behavioral Decision Making, 17, 117-137.

Newell, B. R., \& Shanks, D. R. (2003). Take the best or look at the rest? Factors influencing "one-reason" decision making. Journal of Experimental Psychology: Learning, Memory, and Cognition, 29, 53-65.

Newell, B. R., Weston, N. J., \& Shanks, D. R. (2003). Empirical tests of a fast and frugal heuristic: Not everyone "takes-the-best". Organizational Behavior and Human Decision Processes, 91, 82-96.

Oaksford, M., \& Chater, N. (1998). Rationality in an uncertain world: Essays on the cognitive science of human reasoning. Hove, England: Psychology Press.

Oppenheimer, D. M. (2003). Not so fast! (and not so frugal): Rethinking the recognition heuristic. Cognition, 90, B1-B9.

Over, D. E. (2000a). Ecological issues: A reply to Todd, Fiddick \& Krauss. Thinking and Reasoning, 6, 385-388.

Over, D. E. (2000b). Ecological rationality and its heuristics. Thinking and Reasoning, 6, 182-192.

Rastle, K., Harrington, J., \& Coltheart, M. (2002). 358,534 nonwords: The ARC nonword database. Quarterly Journal of Experimental Psychology, $55 A, 1339-1362$

Seeley, T. D. (2001). Decision making in superorganisms: How collective wisdom arises from the poorly informed masses. In G. Gigerenzer \& R Selten (Eds.), Bounded rationality: The adaptive toolbox (pp. 249-262). Cambridge, MA: MIT Press.

Stanovich, K. E., \& West, R. F. (2002). Individual differences in reasoning: Implications for the rationality debate? In T. Gilovich, D. Griffin, \& D. Kahneman (Eds.), Heuristics and biases: The psychology of intuitive judgment (pp. 421-440). Cambridge, England: Cambridge University Press.

Stanovich, K. E., \& West, R. F. (in press). Evolutionary versus instrumental goals: How evolutionary psychology misconceives human rationality. In D. E. Over (Ed.), Evolution and the psychology of thinking: The debate. Hove, England: Psychology Press.

Todd, P. M., Fiddick, L., \& Krauss, S. (2000). Ecological rationality and its contents. Thinking and Reasoning, 6, 375-384.

Tversky, A., \& Kahneman, D. (1973). Availability: A heuristic for judging frequency and probability. Cognitive Psychology, 4, 207-232.

Received September 2, 2003

Revision received December 10, 2003

Accepted December 27, 2003 